\title{
Review: observational studies adjusting for socioeconomic status and lifestyle show no association between HRT and CAD
}

\author{
Humphrey LL, Chan BK, Sox HC. Postmenopausal hormone replacement therapy and the primary prevention of \\ cardiovascular disease. Ann Intern Med 2002;137:273-84.
QUESTION: What is the effectiveness of postmenopausal hormone replacement therapy (HRT) for primary prevention of cardiovascular disease?

\section{Data sources}

Studies were identified by searching Medline and Cochrane databases from 1966 to December 2000 and by reviewing bibliographies of relevant studies and other publications.

Agency for Healthcare

Research and Quality.

For correspondence:

Dr L L Humphrey,

Veterans Affairs

Medical Center and

Oregon Health and

Science University,

Portland, OR, USA.

humphreyl@ohsu.edu

Abstract and

commentary also

appear in ACP Journal

Club.

Relative risk for coronary artery disease according to use of hormone replacement therapy

(HRT)

\section{Study selection}

Studies were selected if they were randomised controlled trials (RCTs), cohort studies, or case control studies that assessed the use of HRT for primary prevention of cardiovascular disease in postmenopausal women and if English language abstracts were available.

\section{Data extraction}

Data were extracted on study design, type of HRT (unopposed oestrogen or oestrogen plus progestin),

\begin{tabular}{lll} 
& \multicolumn{2}{l}{ Relative risk (95\% credible interval) } \\
\cline { 2 - 3 } Use of HRT & Unadjusted & Adjusted \\
\hline Current & $0.71(0.64$ to 0.78$)$ & $0.97(0.82$ to 1.16$) \ddagger$ \\
\hline Past & $0.78(0.69$ to 0.87$)$ & $1.07(0.90$ to 1.27$) \ddagger$ \\
\hline Ever & Not reported & $1.11(0.84$ to 1.53$) \ddagger$ \\
\hline Any & Not reported & $1.04(0.79$ to 1.44$) \ddagger$ \\
\hline
\end{tabular}

"Includes data from studies that did not adjust for socioeconomic status.

tIncludes data from studies that adjusted for socioeconomic status.

†Not statistically significant. definitions of cardiovascular disease, and potential risk factors included in multivariate models. 2 investigators independently assessed the quality of individual studies. Formal review and meta-analysis were limited to studies of good or fair quality and included RCTs, cohort studies with internal controls, and population based case control studies with $\geq 3$ years of follow up. HRT use was classified as current, past, ever, or any (combined current, past, or ever).

\section{Main results}

Only the results for coronary artery disease (CAD) incidence are reviewed here (3 cohort studies, 9 case control studies, and 1 small RCT). Studies that did not adjust for socioeconomic status (SES) found that current and past use of HRT reduced CAD incidence, whereas studies that adjusted for SES found no association between any measure of HRT use and CAD (table). Similar results were found when analyses were stratified by studies that adjusted for alcohol consumption, exercise, or both.

\section{Conclusions}

Meta-analysis of studies that adjust for socioeconomic status or alcohol consumption and exercise show that current, past, ever, or any use of HRT does not reduce coronary artery disease (CAD). Studies that do not adjust for these factors show a reduced risk for CAD with current and past use.

\section{COMMENTARY}

The 2 meta-analyses by Humphrey $e t a l$ and Nelson $e t a l$, and much of the medical literature, do not clearly define postmenopausal HRT. Although authors often claim that many observational studies show the benefits of HRT on CAD risk, the data almost completely relate to unopposed oestrogen. ${ }^{1}$ Few epidemiological studies have evaluated oestrogen plus progestin administered as continuous combined therapy. A limited number of studies were included in the meta-analyses by Humphrey et al and Nelson $e t$ al, and the included studies sometimes failed to specify type of HRT. Studies published in the $1980 \mathrm{~s}$ and earlier probably refer to unopposed oestrogen therapy.

The lack of specificity in type of HRT is problematic because of the potential for different effects of oestrogen alone and oestrogen plus progestin. It is important to note that the meta-analyses used a common protocol to apply standard criteria for quality ${ }^{2}$ and included only studies of higher quality. For studies assessing CAD outcomes, the authors identified a relation between poorer quality studies and greater protection against CAD.

Changing patterns of use (oestrogen alone replaced by combined oestrogen plus progestin) and indications for use (eg, starting women on treatment before menopause to reduce bone loss) may account, in part, for differences in results. Also, acute effects of combined treatment (eg, the prothrombotic and proinflammatory effects of progestins) may be missed in prospective studies because of lack of attention to early events and follow up.

Data from both epidemiological studies and RCTs of HRT consistently show other thrombotic effects, such as stroke and pulmonary embolus. This argues, at least in part, against a bias related to this pathway in the observational studies. Although socioeconomic status (SES) appears as one other important variable in this analysis, the authors note that the range of cardiovascular risk factors controlled for in observational studies also varied substantially. The authors, however, did not point out that studies that controlled for SES observed similar results before and after such control, which suggests that this is not an explanation for the discrepancy between RCT and observational study results. Is confounding by indication changing over time concurrent with changing patterns of drug combination? Is SES merely a marker for more recent studies that evaluate oestrogen plus progestin? Alternatively, is the timing of exposure in relation to menopause the explanatory factor? Animal studies suggest that oestrogens have beneficial effects in the early stages of atherogenesis, but reduced beneficial effects in the final stages of plaque complications. ${ }^{3}$ 


\title{
Review: risks and benefits of HRT comparing various sources of evidence
}

\author{
Nelson HD, Humphrey LL, Nygren P, et al. Postmenopausal hormone replacement therapy: scientific review.JAMA \\ 2002;288:872-81.

\section{QUESTION: What are the benefits and harms of hormone replacement therapy (HRT) for primary prevention of cardiovascular disease, thromboembolism, osteoporosis, cancer, dementia, and cholecystitis?}

\section{Data sources}

Studies were identified by searching Medline (19662001), HealthSTAR (1975-2001), and the Cochrane Controlled Trials Register; reviewing bibliographies of relevant studies, reviews, and editorials; and contacting experts.

\section{Study selection}

Studies were selected if they included a comparison group of HRT non-users and reported data relating to HRT use and clinical outcomes of interest. Studies were excluded if the sample was selected according to previous events or conditions associated with higher risks of targeted outcomes.

\section{Data extraction}

Data were extracted on study design and type of HRT (unopposed oestrogen or oestrogen plus progestin). 2 reviewers independently assessed study quality as good, fair, or poor using the US Preventive Services Task Force criteria.

\section{Main results}

The findings of the meta-analyses (relative risks) for some outcome categories are summarised in the table with corresponding hazard ratios from the recent Women's Health Initiative (WHI). The results of the meta-analysis showed that HRT reduced the risk of wrist fractures, vertebral fractures, colon cancer, and dementia, and increased the risk of stroke, thromboembolic events, breast cancer, and cholecystitis (table). Findings for some of these outcomes were available from the WHI randomised controlled trial and differed (in terms of statistical significance when using adjusted results for secondary outcomes) for vertebral fractures, colon cancer, coronary heart disease events, and stroke (table).

\section{Conclusions}

Results of this meta-analysis of primarily observational studies and those of a large randomised controlled trial (Women's Health Initiative [WHI]) both show that hormone replacement therapy (HRT) increases risk of thromboembolic events. The meta-analysis shows no effect of HRT on coronary heart disease events, whereas the WHI found an increased risk.
Sources of funding:

Agency for Healthcare Research and Quality and Portland Veterans Affairs Medical Center Women's Health Fellowship.

For correspondence: Dr H D Nelson, Oregon Health and Science

University, Portland,

OR, USA.

nelsonh@ohsu.edu

Abstract and commentary also appear in ACP Journal Club.

Risks associated with ever use of HRT from Nelson et al and from the Women's Health Initiative $[W H I])^{*}$

\begin{tabular}{lll} 
Outcomes & $\begin{array}{l}\text { Relative risk }(95 \% \mathrm{Cl}) \text { from } \\
\text { Nelson et al }\end{array}$ & Hazard ratio (Cl) from WHI \\
Hip fractures & $0.76(0.56$ to 1.01$)$ & $0.66(0.33$ to 1.33$) \ddagger$ \\
\hline Vertebral fractures & $0.60(0.36$ to 0.99$) \dagger$ & $0.66(0.32$ to 1.34$) \ddagger$ \\
\hline Colon cancer & $0.80(0.74$ to 0.86$) \dagger$ & $0.63(0.32$ to 1.24$) \ddagger$ \\
\hline $\begin{array}{l}\text { Coronary heart disease } \\
\text { events }\end{array}$ & $0.91(0.67$ to 1.33$)$ & $1.29(1.02$ to 1.63$) \dagger$ \\
\hline $\begin{array}{l}\text { Stroke } \\
\begin{array}{c}\text { Thromboembolic events } \\
\text { (current use })\end{array}\end{array}$ & $1.12(1.01$ to 1.23$) \dagger$ & $1.41(0.86$ to 2.31$) \ddagger$ \\
\hline Breast cancer $(\geq 5$ y HRT) & 1.23 to 1.35 & $2.11(1.26$ to 3.55$) \dagger \ddagger$ \\
\hline
\end{tabular}

*Abbreviations defined in glossary; meta-analyses based on a random effects model. †Statistically significant. $\ddagger$ Adjusted Cls.

\section{COMMENTARY — continued from previous page}

How can drug formulation change over time and most associations observed in epidemiological studies (ie, breast cancer, pulmonary embolus, stroke, colon cancer, cholecystitis, and osteoporotic fracture) be consistent, and yet CAD outcomes diverge? Consistent evidence across the RCTs argues against chance.

The consistency between non-RCTs and RCTs for non-cardiac outcomes is reassuring and supports other evaluations of the contribution of different study designs to evaluation of medical therapies. ${ }^{4-7}$ Such studies comparing designs may be limited in power, but on average, well designed observational studies show a similar magnitude of estimated benefits as RCTs. We should not focus solely on study design but must also consider the formulation and timing of use of postmenopausal HRT that is being evaluated.

Graham Colditz, MD Channing Laboratory Boston, Massachusetts, USA

1 Stampfer MJ, Colditz GA. Estrogen replacement therapy and coronary heart disease: a quantitative assessment of the epidemiologic evidence. Prev Med 1991;20:4763.

Harris RP, Helfand M, Woolf SH, et al. Current methods of the US Preventive Services Task Force: a review of the process. Am J Prev Med 2001;20:21-35. Mikkola TS, Clarkson TB. Estrogen replacement therapy, atherosclerosis, and vascular function. Cardiovasc Res 2002;53:605-19.

Colditz GA, Miller JN, Mosteller F. How study design affects outcomes in comparisons of therapy. I: Medical. Stat Med 1989;8:441-54

Ioannidis JP, Haidich AB, Pappa M, et al. Comparison of evidence of treatment effects in randomized and nonrandomized studies. JAMA 2001;286:821-30.

Benson K, Hartz AJ. A comparison of observational studies and randomized, controlled trials. N Engl J Med 2000;342:1878-86.

Concato J, Shah N, Horwitz RI. Randomized, controlled trials, observational studies, and the hierarchy of research designs. N Engl J Med 2000;342:1887-92. 